\title{
Association of interleukin 17 / angiotensin II with refractory hypertension risk in hemodialysis patients.
}

\author{
Zebin Wang ${ }^{1,2,3}$, Wei Shi ${ }^{2}$, Xinling Liang ${ }^{2}$, Wenjian Wang ${ }^{2}$, Jianbo Liang ${ }^{3}$
}

1. Southern Medical University, Guangzhou, China

2. Department of Nephrology, Guangdong General Hospital, Guangdong Academy of Medical Hospital, Guangzhou, China

3. Department of Nephrology, The Second Affiliated Hospital of GuangZhou

Medical University, Guangzhou, China

\begin{abstract}
Objective: The study was performed to investigate the association of interleukin 17 (IL 17) or angiotensin II (Ang II) with refractory hypertension risk in hemodialysis patients.

Methods: Ninety hemodialysis patients were enrolled into this study, and those with hypertension were divided into two groups. The Easy-to-Control Hypertension group (ECHG) had fifty patients, while the refractory hypertension group (RHG) had forty patients. Twenty healthy individuals were recruited as the control group. IL17 and Ang II were determined using a human IL 17 / Ang II enzyme-linked immunosorbent assay kit. Serum IL 17 and Ang II concentrations in RHG patients were higher than those in ECHG patients.

Results: Serum IL 17 and Ang II concentrations in both patient groups were higher than those in the control group. Linear regression analysis showed a positive correlation between IL 17 and Ang II. In multivariate regression analysis, we found that IL17 and Ang II were associated with refractory hypertension risk in hemodialysis patients.

Conclusion: IL17 and Ang II were associated with refractory hypertension risk in hemodialysis patients. There was also a positive correlation between IL 17and Ang II.

Keywords: Interleukin 17, Angiotensin II, refractory hypertension, hemodialysis

DOI: http://dx.doi.org/10.4314/ahs.v16i3.17

Cite as: Wang Z, Shi W, Liang X, Wang W, Liang J. Association of interleukin 17 / angiotensin II with refractory hypertension risk in hemodialysis patients. DOI: http://dx.doi.org/10.4314/ahs.v16i3.17
\end{abstract}

\section{Introduction}

Patients with End State Renal Disease (ESRD) present very late and with cardiovascular complications ${ }^{1}$. Furthermore, patients with ESRD who are going for dialysis tend to have refractory hypertension, anemia, and other complications, ${ }^{2,3}$. Many ESRD patients have to take several kinds of anti-hypertensive drugs, but even when they receive the maximum permissible doses; it remains difficult to treat their hypertension. Currently, about $70 \%$ of hemodialysis patients fail to have their
Corresponding author:
Wei Shi, Department of Nephrology,
Guangdong General Hospital,
Guangdong Academy of Medical
Hospital, Guangzhou, China,
E-mail: 13822182216@139.com

African Health Sciences Vol 16 Issue 3, September 2016 hypertension controlled ${ }^{2}$, and there is increased mortality and cardiovascular and cerebrovascular morbidity among them ${ }^{4}$. Activation of the Renin-Angiotensin-Aldosterone System (RAAS) and hypervolaemia are often found and considered to be the main causes of refractory hypertension in Chronic Kidney Disease (CKD) patients $^{5,6}$. However, blocking the RAAS, and controlling the volume of blood are not good approaches to treating the refractory hypertension well. Angiotensin II (Ang II) is the major effect messenger of the RAAS and exhibits a wide range of responses, for instance induction of the accumulation and deposition of collagen $^{7}$.

Interleukin 17 (IL 17), with a molecular weight of $35 \mathrm{kDa}$, is a type of homodimeric glycoprotein containing 155 amino acids and is generated by Th17 cells ${ }^{8}$. IL 17 is a critical factor in assisting mammals to defend against a wide range of pathogens ${ }^{8,9}$. Angiotensin II (Ang II) can 
stimulate Th17 cells to generate IL17. Madhur et $\mathrm{al}^{10}$ discovered that IL17 is important in the development of hypertension. Serum IL 17 level in hypertension patients is much higher than that in normal individuals, and IL 17 concentration is positively correlated with blood pressure values. Interestingly, Chung et $\mathrm{al}^{11}$ found that hemodialysis patients had an evidently increased IL 17 level, compared to healthy people in the control group, and IL17 could not be removed from the body by hemodialysis. Therefore, IL 17 could play a role in the causation of refractory hypertension in hemodialysis. This study was performed to assess the association between IL 17, angiotensin II and refractory hypertension risk in hemodialysis patients.

\section{Methods}

This study was performed in the Department of $\mathrm{Ne}$ phrology, the Second Affiliated Hospital of GuangZhou Medical University, GuangZhou, China. From December 2012 to December 2014, 90 hemodialysis patients were enrolled into the study, and they were newly treated with steady hemodialysis for at least three months. Exclusion criteria included the presence of inflammatory disease, malignancy, chronic liver disease, chronic obstructive airway disease, chronic rheumatic heart disease, congenital heart disease, and those patients aged below 18 years or above 70 .

The causes of ESRD included chronic glomerulonephritis in 35 patients, diabetic nephropathy in 27 patients, hypertensive nephrosclerosis in 20 patients, obstructive uropathy in 3 patients, tubulointerstitial nephritis in 3 patients, and unknown etiology in 2 patients. Fifty patients were put into the easy-to-control hypertension group (ECPG), while forty others were put into the refractory hypertension group (RHG). Twenty healthy individuals were used as the control group in order to define basal IL 17 level. The study was approved by the University ethics committee, and written informed consent was obtained from all patients and healthy individuals before they joined the study.

Supine blood pressure (BP) was measured three times with a mercury sphygmomanometer, and the first and fifth Korotokoff sounds were taken as systolic and diastolic. Mean arterial pressure (MAP) was calculated as diastolic $\mathrm{BP}+1 / 3$ (systolic BP-diastolic BP). Patients' systolic $\mathrm{BP}<140 \mathrm{mmHg}$ and diastolic $\mathrm{BP}<90 \mathrm{mmHg}$ were considered to have attained the goal of anti-hypertension treatment. Refractory hypertension was defined as blood pressure that was above the goal in spite of the concurrent use of three antihypertensive agents given in appropriate doses of different classes for at least three months 12 .

The blood samples were drawn before dialysis. Fasting venous blood was collected for the measurement of high sensitivity CRP (hs-CRP), serum albumin, calcium, phosphorus, Parathyroid hormone (PTH), cholesterol (CHOL), triglycercide (TG), low-density lipoprotein (LDL), and high-density lipoprotein (HDL). IL 17 and Ang II were determined using human Enzyme-Linked Immunosorbent Assay (ELISA) kits (Shanghai Lengton Bioscience Co., Shanghai, China). All specimens were measured in triplicate.

Total weekly urea clearance $(\mathrm{Kt} / \mathrm{V})$ was measured to assess the adequacy of dialysis. ACE inhibitor, ARB, $\beta$-blocker, calcium channel clocker, or $\alpha$-blocker were used to control the patients' hypertension, and all hemodialysis patients received two or more antihypertensive drugs including ACE inhibitor or ARB.

\section{Statistical analysis}

Data was expressed as means \pm SD (standard deviation). Baseline characteristics of hemodialysis patients in the two groups were compared using Analysis Of Variance (ANOVA) for continuous data and chi-square analyses for categorical data. Comparisons of the IL 17 expression among the control, ECHG, and RHG group, were performed using one-way ANOVA for continuous variables and X2 statistics for differences in proportion. Multivariate regression analyses were done to evaluate the relationships between IL 17, Ang II and blood pressure. A p value $<0.05$ was considered statistically significant.

\section{Results}

\section{Baseline characteristics of patients in the two hemo- dialysis patients groups}

Baseline characteristics of the ECHG group and RHG group are shown in Table 1 . The average age was $55.30 \pm 9.25$ years in the ECHG group and $54.47 \pm 9.26$ years in the RHG group. The ratio of males to females was 26:24 and 24:16 in the ECHG and RHG group re- 
spectively. There was no difference in the incidence of diabetes, hemoglobin, albumin, hsCPR, calcium, phosphorus, and lipoprotein between the two groups. IL 17 and Ang II concentrations were $10.88 \pm 1.41 \mathrm{pg} / \mathrm{m}$ land $89.09 \pm 24.03 \mathrm{ng} / \mathrm{L}$ in the ECHG group; and $14.51 \pm 1.43$ $\mathrm{pg} / \mathrm{ml}$ and $118.02 \pm 12.21 \mathrm{ng} / \mathrm{L}$ in the RHG group. There was difference between two groups $(\mathrm{p}<0.01$, Table 1$)$.

Table 1. Clinical characteristics of patients between ECHG group and RHG group

\begin{tabular}{llll}
\hline & ECHG & RHG & $P$ value \\
\hline Age $($ year $)$ & $55.30 \pm 9.25$ & $54.47 \pm 9.26$ & 0.676 \\
Male/female & $26 / 24$ & $24 / 16$ & 0.450 \\
Diabetes incidence & 12 & 10 & 0.913 \\
Systolic pressure & $120.18 \pm 15.07$ & $159.37 \pm 18.05$ & 0.000 \\
Diastolic pressure & $73.00 \pm 10.79$ & $98.05 \pm 9.96$ & 0.000 \\
Mean arterial pressure (MAP) & $88.72 \pm 12.07$ & $118.49 \pm 11.30$ & 0.000 \\
BMI $(\mathrm{kg} / \mathrm{m} 2)$ & $23.10 \pm 3.61$ & $23.59 \pm 3.63$ & 0.524 \\
$\mathrm{Hb}(\mathrm{g} / \mathrm{L})$ & $116.10 \pm 22.15$ & $111.30 \pm 20.44$ & 0.421 \\
$\mathrm{ALB}(\mathrm{g} / \mathrm{L})$ & $36.02 \pm 5.17$ & $35.88 \pm 5.45$ & 0.901 \\
$\mathrm{hsCRP}(\mathrm{mg} / \mathrm{L})$ & $3.50(0.87,8.9)$ & $3.52(0.87,8.85)$ & 0.803 \\
$\mathrm{iPTH}(\mathrm{pg} / \mathrm{L})$ & $30.56 \pm 50.41$ & $33.34 \pm 55.88$ & 0.809 \\
$\mathrm{Ca}(\mathrm{mmol} / \mathrm{L})$ & $2.29 \pm 0.28$ & $2.30 \pm 0.28$ & 0.903 \\
$\mathrm{P}(\mathrm{mmol} / \mathrm{L})$ & $1.55 \pm 0.42$ & $1.57 \pm 0.43$ & 0.788 \\
TC $(\mathrm{mmol} / \mathrm{L})$ & $4.77 \pm 1.12$ & $4.67 \pm 1.09$ & 0.672 \\
TG $(\mathrm{mmol} / \mathrm{L})$ & $3.34 \pm 8.17$ & $3.50 \pm 9.11$ & 0.930 \\
$\mathrm{HDL}(\mathrm{mmol} / \mathrm{L})$ & $0.98 \pm 0.25$ & $0.98 \pm 0.26$ & 0.960 \\
$\mathrm{LDL}(\mathrm{mmol} / \mathrm{L})$ & $2.46 \pm 0.78$ & $2.55 \pm 0.79$ & 0.565 \\
$\mathrm{IL} 17(\mathrm{pg} / \mathrm{ml})$ & $10.88 \pm 1.41$ & $14.51 \pm 1.43$ & 0.000 \\
Angiotensin II(ng/L) & $89.09 \pm 24.03$ & $118.02 \pm 12.21$ & 0.000 \\
eGFR $(\mathrm{ml} / \mathrm{min})$ & $9.63 \pm 3.73$ & $9.54 \pm 3.95$ & 0.912 \\
$\mathrm{Kt} / \mathrm{v} \quad$ & $1.3 \pm 0.12$ & $1.3 \pm 0.04$ & 0.860 \\
\hline
\end{tabular}

Notes: ECHG: easy-to-control hypertension group; RHG: refractory hypertension group; BMI: body mass index; eGFR: estimated glomerular filtration rate

The relationships between IL17, Ang II, and MAP Linear regression analysis showed a positive linear correlation between IL 17 and Ang II, with a regression coefficient (B) of 7.617(95\%CI 6.969-8.264), $\mathrm{t}=23.375$, $\mathrm{p}<0.01, \mathrm{R} 2=0.861($ Fig $1 \mathrm{~A})$.
IL 17 and MAP also had a positive linear correlation, with a regression coefficient (B) of 0.081 (95\%CI 0.0710.092), $\mathrm{t}=15.709, \mathrm{p}<0.01, \mathrm{R} 2=0.737$ (Fig $1 \mathrm{~B}$ ). 


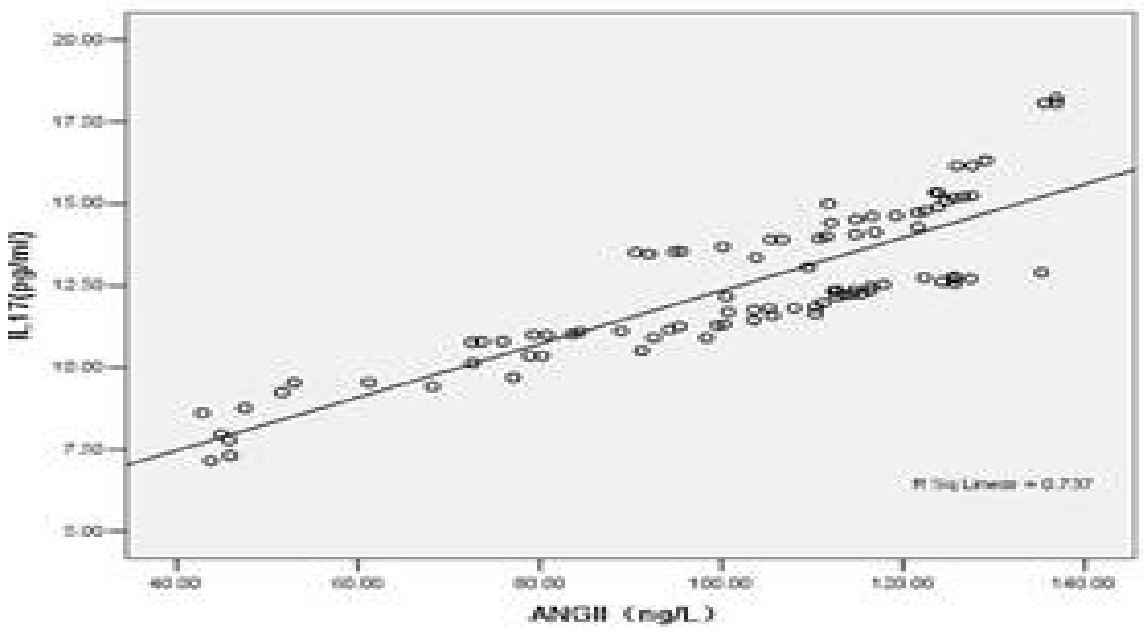

A

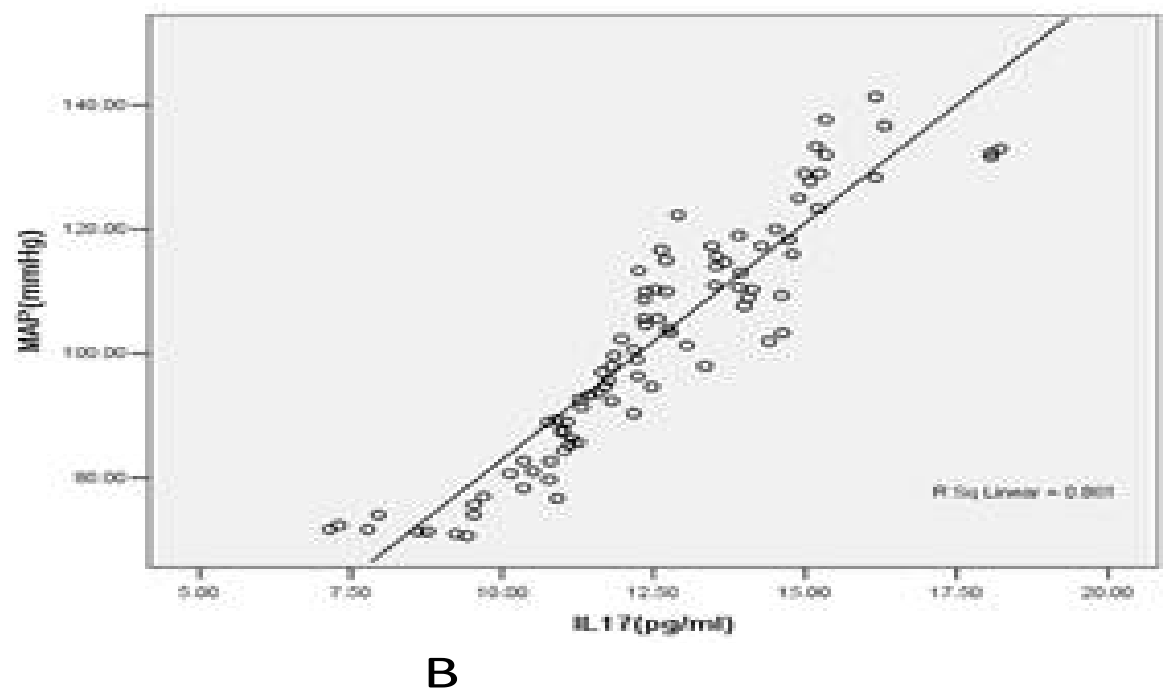

Fig 1. Linear regression analysis showed linear correlation between angiotensin II, IL 17 and MAP. A. Linear regression analysis showed positive linear correlation between angiotensin II and IL 17, the regression coefficient(B) was $7.617\left(95 \% \mathrm{Cl}\right.$ 6.969-8.264), $t=23.375, p<0.01, \mathrm{R}^{2}=0.861$. B. Linear regression analysis showed positive linear correlation between IL 17 and MPA, the regression co-efficient(B) was $0.081\left(95 \% \mathrm{Cl}\right.$ 0.071-0.092), $t=15.709, p<0.01, R^{2}=0.737$.

In the multiple linear regression analysis for MAP, serum IL 17 (B=6.32, $\mathrm{t}=10.221, \mathrm{P}<0.01)$ and Ang II $(\mathrm{B}=0.143$, $\mathrm{t}=2.442, \mathrm{P}=0.017)$ had significant correlation with MAP after adjusting for age, diabetes, BMI, hemoglobin, hsCRP, eGFR, and Kt/v. 


\section{Table 2 Multiple linear regression analysis showing relationship between IL 17, Ang II and mean arterial pressure in hemodialysis patients.}

\begin{tabular}{llll}
\hline Variables & $B$ & $t$ value & $P$ value \\
\hline IL 17 & 6.320 & 10.221 & 0.000 \\
Ang II & 0.143 & 2.442 & 0.017 \\
\hline
\end{tabular}

\section{Discussion}

This study aimed to investigate the association between IL 17 and the risk of refractory hypertension in hemodialysis patients. We found that increased IL 17 was associated with the risk of refractory hypertension in hemodialysis patients. We did not find any other study reporting this relationship. However, our study had a small sample size. More studies with larger sample sizes could be performed to confirm these findings.

Interestingly, we also found that the increased Ang II was associated with the risk of refractory hypertension in hemodialysis patients. A number of previous studies report that the increased Ang II was associated with the hypertension, and ACE inhibitor, ARB could reduce the value of $\mathrm{BP}^{13-15}$. In this study, we reported a positive correlation between Ang II and IL 17. However, the mechanism should be assessed more in the future.

The most common finding in dialysis patients with refractory hypertension is volume overload. In this study, the volume overload was controlled for all the patients. However, since it was an observational study, we could not answer this question that dialysis patients with refractory hypertension have volume overload.

In conclusion, we found that IL17 and Ang II were associated with refractory hypertension risk in hemodialysis patients, and there was a positive correlation between IL 17 and Ang II.

\section{Acknowledgements}

None.

\section{Conflict of interest}

The authors declare that they have no conflict of interest.

\section{References}

1. Tumwine JK. From renal and cardiovascular disease risk, to reproductive health, increasing violence in Nigeria and use of ultra sound in blunt trauma. Afr Health Sci 2006, 6(3):131.

2. Agarwal R, Nissenson AR, Batlle D, Coyne DW, Trout JR, Warnock DG. Prevalence, treatment, and control of hypertension in chronic hemodialysis patients in the United States. Am J Med 2003, 115(4):291-297.

3. Kaze FF, Kengne AP, Mambap AT, Halle MP, Mbanya D, Ashuntantang G. Anemia in patients on chronic hemodialysis in Cameroon: prevalence, characteristics and management in low resources setting. Afr Health Sci 2015, 15(1):253-260.

4. Foley RN, Parfrey PS, Harnett JD, Kent GM, Murray DC, Barre PE. Impact of hypertension on cardiomyopathy, morbidity and mortality in end-stage renal disease. Kidney Int 1996, 49(5):1379-1385.

5. Campese VM, Mitra N, Sandee D. Hypertension in renal parenchymal disease: why is it so resistant to treatment? Kidney Int 2006, 69(6):967-973.

6. Borrelli S, De Nicola L, Stanzione G, Conte G, Minutolo R. Resistant hypertension in nondialysis chronic kidney disease. Int J Hypertens 2013, 2013:929183.

7. Zhou TB, Ou C, Rong L, Drummen GP. Effect of all-trans retinoic acid treatment on prohibitin and reninangiotensin-aldosterone system expression in hypoxiainduced renal tubular epithelial cell injury. J Renin Angiotensin Aldosterone Syst 2014, 15(3):243-249.

8. Kolls JK, Linden A. Interleukin-17 family members and inflammation. Immunity 2004, 21(4):467-476.

9. Ivanov S, Linden A. Th-17 cells in the lungs? Expert Rev Respir Med 2007, 1(2):279-293.

10. Madhur MS, Lob HE, McCann LA, Iwakura Y, Blinder Y, Guzik TJ, Harrison DG. Interleukin 17 promotes angiotensin II-induced hypertension and vascular dysfunction. Hypertension 2010, 55(2):500-507. 
11. Chung BH, Kim KW, Sun IO, Choi SR, Park HS, Jeon EJ, Kim BM, Choi BS, Park CW, Kim YS et al. Increased interleukin-17 producing effector memory T cells in the end-stage renal disease patients. Immunol Lett 2012, 141(2):181-189.

12. Calhoun DA, Jones D, Textor S, Goff DC, Murphy TP, Toto RD, White A, Cushman WC, White W, Sica D et al. Resistant hypertension: diagnosis, evaluation, and treatment: a scientific statement from the American Heart Association Professional Education Committee of the Council for High Blood Pressure Research. Circulation 2008, 117(25):e510-526.
13. Matsuyama N, Tsutsumi T, Kubota N, Nakajima T, Suzuki H, Takeyama Y. Direct action of an angiotensin II receptor blocker on angiotensin II-induced left atrial conduction delay in spontaneously hypertensive rats. $\mathrm{Hy}$ pertens Res 2009, 32(8):721-726.

14. Miyauchi K, Yamazaki T, Watada H, Tanaka Y, Kawamori R, Imai Y, Ikeda S, Kitagawa A, Ono Y, Murayama $\mathrm{F}$ et al. Management of home blood pressure by amlodipine combined with angiotensin II receptor blocker in type 2 diabetes. Circ J 2012, 76(9):2159-2166. 15. Postnov Iu V. Ene 